\title{
Carnets
}

Revue électronique d'études françaises de l'APEF

Deuxième série - 16 | 2019

Le Récit inachevé: études sur Mai 68

\section{La génération Mai 68 revisitée dans deux romans français contemporains}

Les Images d'Alain Rémond et Camarades de classe de Didier Daeninckx José Domingues de Almeida

\section{OpenEdition}

\section{Journals}

Édition électronique

URL : http://journals.openedition.org/carnets/9789

DOI : $10.4000 /$ carnets.9789

ISSN : 1646-7698

Éditeur

APEF

Référence électronique

José Domingues de Almeida, «La génération Mai 68 revisitée dans deux romans français

contemporains », Carnets [En ligne], Deuxième série - 16 | 2019, mis en ligne le 31 mai 2019, consulté

le 08 novembre 2019. URL : http://journals.openedition.org/carnets/9789 ; DOI : 10.4000/carnets. 9789

Ce document a été généré automatiquement le 8 novembre 2019.

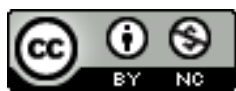

Carnets est mis à disposition selon les termes de la licence Creative Commons - Atribution - Pas d'utilisation commerciale 4.0 International. 


\section{La génération Mai 68 revisitée dans deux romans français contemporains}

Les Images d'Alain Rémond et Camarades de classe de Didier Daeninckx

José Domingues de Almeida

Cet été 68 était loin, bien loin, mais il gardait un goût de commencement. Et c'était là qu'il voulait tout recommencer (Les Images, p. 115)

Sur quels récifs ces rêves d'alors s'étaient-ils fracassés? (Camarades de classe, p. 107)

1 Comme le rappelle Ursula Gauthier dans un éditorial intitulé «Une extase de l'Histoire »: "De Mai 68, on a tout dit et son contraire. Cet événement inclassable a suscité une pléthore d'interprétations, du simple malaise de l'université à la crise de civilisation planétaire, en passant par l'entreprise de subversion politique, la révolte festive des jeunes, la fuite dans l'hédonisme - ou le nihilisme. Ou encore une parenthèse utopique, ou un conflit de classe d'un type nouveau (Gauthier, 2018:3), « (...) à la fois libertaire et égalitaire » (ibid.).

2 Aussi, les retombées de Mai 68, et ce contrairement à la Révolution des Cillets, ne ressortissent-elles pas vraiment à la commémoration officielle et consensuelle, raison pour laquelle Emmanuel Macron a finalement renoncé à un projet célébratif à portée nationale. Elles s'inscrivent plutôt dans une logique de bilan sociétal diffus, sans cesse réévalué ou réapproprié, ce que Henri Weber désigne tantôt par « le bilan de mai ", tantôt par "l'interprétation de mai », ou encore "le travail de mai » (Weber, 2008: 121-155). Pour ce dirigeant du mouvement de Mai 68, ancien dirigeant du Parti Socialiste et député européen, il s'agissait tous les dix ans, dans les rééditions évocatoires, de sauver l'honneur et l'héritage soixante-huitards bafoués par les multiples attaques imputant à Mai 68 l'origine des impasses et des inadaptations hexagonales. 
D'une part, il y a la pensée et l'essai qui, ces vingt dernières années, se sont mis à interroger Mai 68 dans ses slogans, ses mouvements et sa morale, souvent pour essayer de justifier le déclin de la culture et des valeurs républicaines. L'essai La Pensée 68. Essai sur l'anti-humanisme contemporain de Luc Ferry et Alain Renaut (1988) signale une critique sans concession de la philosophie des années 68 accusée d'accoucher de paradoxes improbables, voire antagoniques (ibid. : 82-83).

4 Cette tendance, qui associe humanisme et individualisme à outrance, trouve un relai dans la lecture sociologique de Gilles Lipovetsky, surtout depuis la parution de L'Ère $d u$ vide (1988), étant donné que cet auteur impute à Mai 68 la seconde révolution individualiste, le " processus de personnalisation » en cours depuis lors qui aurait déjà causé d'irréversibles dégâts dans l'idéal moderne de subordination de l'individuel aux règles rationnelles collectives en désamorçant un hyper-individualisme diffus, indifférent, hédoniste et narcissique.

Or, c'est la traduction de ce discours dans l'argumentaire politique

hexagonal qui choque Henri Weber, notamment dans la fameuse attaque de Nicolas Sarkozy aux fruits sociétaux de Mai 68. Dans son discours à l'université d'été des "Jeunes populaires" à Marseille en septembre 2006, l'ancien président de la République en appelait à une liquidation totale des prétendus acquis de Mai 68 et de son "travail » de sape des assises républicaines qui, d'après lui, auraient empêché la croissance, détruit l'école, incité le communautarisme et nivelé toutes les instances symboliques dans un relativisme qui mine l'autorité de l'État et compromet le développement économique de la France face à ses principaux partenaires commerciaux (Weber, 2008 : I-XXI).

6 À cet égard, plusieurs mesures programmatiques sarkoziennes, ou même un certain discours macroniste s'inscrivent dans une logique discursive de souci de restauration d'une France qui doit renouer avec une grandeur perdue quelque part à la faveur de ce qu'Edgar Morin désigne par «(...) la tradition insurrectionnelle de la France (...)» (Morin, $2018: 8$ ).

7 Cet argumentaire, qu'il s'agit de ne pas jeter avec l'eau du bain, comme le fait Weber par antagonisme politique et idéologique, va jusqu'à exhumer une fracture générationnelle en reprochant à l'égoïsme des soixante-huitards, aujourd'hui retraités hédonistes et gâtés, la précarité dont les jeunes générations payent les frais, comme si c'était là une facture à honorer maintenant, par des acteurs qui n'ont certes pas vécu Mai 68.

8 Ce courant de pensée a produit, pour reprendre Henri Weber, «tout une littérature dépei[gnant] aujourd'hui les ravages provoqués par la lame de fond féministe [et soixante-huitarde]: couples brisés, enfants déboussolés, succès professionnels mais déserts affectifs ; visibilité sociale, mais solitude » (Weber, 2008 : 176). Inconsciemment, Weber pointe du doigt les écrivains que Nancy Huston devait désigner par «Professeurs de désespoir » (2004), ces nouveaux réactionnaires qui s'acharnent, par la fiction narrative, à « liquider le travail de mai », comme c'est le cas de Michel Houellebecq.

9 Aussi peut-on approcher l'épiphénomène de Mai 68 dans ses retombées contradictoires à partir d'un échantillonnage fictionnel. Une simple recherche sur le moteur Google à partir des mots-clés « roman » et « Mai 68 » fait immédiatement apparaittre une liste de 
vingt-neuf titres français ${ }^{1}$ censés se référer plus ou moins allusivement à cet événement social majeur de l'histoire française et occidentale.

Dans un récent numéro thématique spécial du Nouveau Magazine Littéraire sur Mai 68, Chloé Brendlé met en évidence "L'introuvable récit» (Brendlé, 2018: 46) de cette époque avant de se demander: "Mais où sont donc passés les jours de Mai? Qui se souvient de L'Irrévolution de Pascal Lainé, prix Médicis en 1971 ? Qui lit encore Derrière la vitre, de Robert Merle, retraçant une journée dans la vie d'étudiants de Nanterre, celle du 22 mars 1968 ?» (ibid.). Et Brendlé de regretter que «D'emblée, entre le printemps 1968 et la littérature, c'est une sorte de rendez-vous manqué, comme si tout le désir d'écrire s'était déversé dans la rue, sur les murs, ensemble, en cris, et comme si après coup il devenait vain de saisir le surgissement collectif sinon sous la trace de réminiscences mélancoliques» (ibid.), de sorte qu'il faudrait plutôt parler de "Docufiction édifiante», "style saga» (ibid.: 47). Parmi ces tentatives de reconstitution, de filiation ou de distillation de Mai 68, la critique cite «(...) Didier Daeninckx et ses Camarades de classe (2008)» (ibid.: 47). Pour notre étude, nous partirons justement de ce roman auquel nous ajouterons Les Images d'Alain Rémond (1997).

11 Ces deux récits n'exhument qu'allusivement les événements historiques du long printemps parisien et français de 1968 en suivant des destins d'ex-soixante-huitards reconvertis ou mal à l'aise dans d'autres activités ou convictions. Dans Les Images - écrit cette fois à l'imparfait et non plus au conditionnel présent comme dans Les Choses (1965) - Rémond imagine une suite narrative pour le couple de psychosociologues consumériste créé par Georges Perec. Les jeunes et dynamiques Jérôme et Sylvie ont quitté le Paris des années soixante pour diriger une agence de publicité à Bordeaux : «Ils n'avaient pas trente ans. Ils avaient été de vagues étudiants, de vagues sociologues, de vagues sondeurs. Ils n'étaient pas préoccupés par leur avenir, ils pensaient que les choses arriveraient, qu'elles leur seraient données, quand il le faudrait » (Rémond, 1997 : 9). Ils veulent réussir dans la vie. Or, en province, ils ont du mal à masquer leur parisianisme.

Ils s'étaient pourtant redécouverts parisiens avec une pointe d'envie, un an après leur mue provinciale, en mai 68 . Vexés, ils avaient trouvé, comme des enfants, que ce n'était pas juste: pourquoi fallait-il que Paris se réveille, délire, s'embrase, flamboie, juste après leur départ? Tous leurs amis devaient être au cœur de l'action, dans les manifestations, à l'odéon, alors qu'eux-mêmes étaient condamnés à ne vivre toute cette effervescence que par procuration, dans une sorte d'exil (ibid. $: 15)$.

12 Dans la foulée de Mai 1968, ils deviennent des publicitaires à la mode, des gourous des ventes commerciales. Imprévisiblement, ils rentrent à Paris, et les voilà mêlés à l'évolution profonde du panorama audiovisuel français des années soixante aux années quatre-vingt-dix. Nous sommes à l'ère des conseillers en communication, des entreprises commanditaires, du démantèlement et privatisation des chaînes privées et de la téléréalité généralisée (ibid. : 74, 79) fondée sur l'audimat (ibid.: 76). Bref, le cheminement de bien des soixante-huitards à la faveur de la subtile transition des choses vers le royaume des images, même si cette évolution n'est nullement incompatible.

13 Dans Camarades de classe de Didier Daeninckx (2008), la narratrice, une certaine Dominique, travaille également dans une agence de publicité, alors que François, son mari - qui comme elle approche la soixantaine - est cadre dans un groupe 
pharmaceutique en cours de restructuration, et vit la déprime à l'idée de se voir viré. Or, un jour, Dominique intercepte un message destiné à François à partir d'un forum de rencontre d'anciens camarades de classe - camarades-de-classe.com - provenant d'un ancien ami de lycée qui tente de renouer le contact grâce audit forum. À l'insu de François, Dominique répond, entre dans les échanges et dans les confidences de ces anciens élèves de la classe de 3e du collège Gabriel Péri d'Aubervilliers, ravivés à partir d'une photo de visite d'études postée pour rappeler l'ambiance de la banlieue ouvrière et communiste parisienne... On apprendra à la fin que Dominique est, elle aussi, un camarade, un ancien élève qui a subi une opération transgenre. Or les différents courriels échangés révèlent des conflits et des malaises qui couvent dans le bouillon référentiel de Mai 68, et surtout des années soixante-dix.

En fait, dans les deux romans, Mai 68 s'avère, comme le caractérise Edgar Morin, « (...) une brèche sous la ligne de flottaison de l'ordre social, par laquelle se sont engouffrées des valeurs, des aspirations, des idées nouvelles, appelées à transformer en profondeur notre civilisation» (Morin, 2018: 10); «une faille», pour reprendre Michel de Bris (2018: 51), raison pour laquelle cet événement ne marque pas seulement ceux qui l'ont fait ou vécu. Comme le signale Diane Lisarelli dans «Je n'ai pas fait 68 mais... », il serait de l'ordre du « temps vertical », du « temps en suspens » (Lisarelli, 2018:40-41); c'est-à-dire pris comme référence, voire récupérable; en tous cas dont le sens est inépuisable.

Or ces deux récits dégagent des perspectives pertinentes sur l'évolution et la reconversion, voire le reniement des générations de soixante-huitards, curieusement passés à l'empire de l'image. Si dans le cas de Les Images, la diégèse doit beaucoup au parcours personnel de l'auteur, Alain Rémond, un habitué de l'audiovisuel français, elle expose dans les deux cas une fascination de l'image et de la publicité comme traits d'une société en évolution. En effet, Jérôme et Sylvie se sont reconvertis dans la publicité : «Elle [la publicité] était écologiste, féministe, libertaire, peace and love, contestataire, antiraciste, elle respectait les droits du consommateur et ceux des citoyens. Jérôme et Sylvie vendaient des nouilles. Mais c'étaient des nouilles libres " (Rémond, 1997 : 22), et ils se sont enrichis (ibid. : 23).

De même, dans Camarades de classe, Dominique a fait carrière dans les campagnes publicitaires et de communication. Elle dirige une équipe assez dynamique et agressive dans cette activité (Daeninckx, 2008 : 47, 118-119). Cette tendance semble accréditer le jugement sévère de Luc Ferry, qui encore récemment ne démordait pas de ses anciennes critiques, voire les confirmait. Il prétendait que «(...) c'était une société hyperlibérale qui se profilait» (ibid. : 44), tout en rappelant que "Quant aux soixantehuitards, à quelques très rares exceptions près, ils vont se reconvertir dans la pub, le cinéma, l'entreprise, voire au Sénat, dans l'inspection générale et dans la socialdémocratie (...)» (ibid. : 45).

Dans ces deux romans, Mai 68 s'avère le repère historique et le contexte social à partir duquel le lecteur peut mesurer l'évolution de la France à travers le parcours des personnages. Dans Les Images, le narrateur extradiégétique recourt souvent à la parenthèse réflexive pour tisser ses considérations sur le moment que le pays est en train de vivre, avant d'en caractériser les inévitables conséquences sociétales :

Ce que mettait en cause Mai 68, c'était, entre autres, l'aliénation des masses par la publicité, leur manipulation par des slogans débiles, leur abrutissement par des réflexes conditionnés. Or, travaillant eux-mêmes dans la publicité, ils étaient, 
objectivement, des agents de l'aliénation. Comment pouvaient-ils être des deux côtés de la barricade, fustiger ce qu'ils faisaient et qu'ils aimaient faire ? (ibid. : 16). l'audiovisuel des aspirations soixante-huitardes, ce sur quoi Pascal Ory reviendra pour contextualiser l'application, selon lui, d'une "logique libérale » (Ory, 2018: 71) au panorama de l'ancienne ORTF. Ainsi, Jérôme incarne cette évolution et le triomphe de l'image du fait de son parcours et de son ascension personnels au sein de l'univers télévisuel : « Mais au fur et à mesure qu'ils s'imposaient dans la publicité, ils avaient commencé à prendre la télévision de plus en plus au sérieux » (Rémond, 1997 : 25), et mesuré son «impact socioculturel» (ibid.) «(...) en ces années soixante-dix si furieusement idéologiques» (ibid. : 27) qui voient la promotion à l'écran de «jeux idiots » (ibid. : 31, 42). Mais les années quatre-vingt amorcent un nouveau tournant dans la télévision. En effet, on commence à traiter de l'image des hommes politiques (ibid.: 44), à fabriquer de toutes pièces des acteurs politiques, ce qui suscitera l'éloignement de Sylvie, de plus en plus déçue par la compromission de Jérôme, et la première crise de conscience de ce désormais «consultant» (ibid.: 51): "Mais que voulait Jérôme ? Il ne savait plus très bien » (ibid. : 45). Le voilà de plus en plus pris de «scrupules» (ibid.) devant un processus irrépressible en marche : «(...) il faut en finir avec la télé de papa » (ibid. : 51, 72). Jérôme a petit à petit mauvaise conscience (ibid. : $82,86,91)$.

Alors, plus rien n'est défendu à l'écran : «Plus rien n'était tabou. À commencer par l'argent, le succès, l'ambition, la compétition » (ibid. : 52). Les années quatre-vingt-dix voient l'arrivée des «studios high-tech» (ibid. : 59) et l'omniprésence de fascinants «mur[s] d'images» (ibid. : 91, 93) dans ces studios incarnés par le style CNN. Ce « hold up des images sur la réalité » (ibid.: 96) n'est pas sans évoquer l'hyper-réalité baudrillardienne (1981), d'autant plus que la télévision rejoint progressivement le « simulacre » (Rémond, $1997:$ 105-106).

Bien sûr, il est impossible de séparer totalement les réflexions du narrateur de la pensée de l'auteur Alain Rémond, fin connaisseur de l'audiovisuel français :

Il avait dirigé une agence de pub, il avait été l'un des plus brillants créatifs de sa génération, il avait conseillé un ministre en mal d'image, il avait rejoint le monde de la télévision, il avait débattu, colloqué jusqu'à plus soif (...). En réalité, Jérôme avait passé sa vie à une seule chose : à essayer de trouver sa place dans le monde des images. Il avait théorisé, conseillé, pratiqué puis dénoncé (ibid. : 112).

D'autant plus que le caractère subi, voire imposé, de cette évolution se voit renforcé par certains éléments narratifs qui ponctuent et structurent le récit, comme le « on » impersonnel récurrent : «On lui expliqua (...)» (ibid. : 81), et amplifié par les bornes chronologiques balisant narrativement la rapide évolution du récit et de la télévision française : « Un soir » (ibid. : 47), « Un jour » (ibid. : 55).

À cet égard, le narrateur insiste sur les nouveaux concepts en vogue dans l'audiovisuel : « challenge " (ibid. : 69), « rentabilisation» et "privatisation» (ibid.: 71) qui ont marqué l'histoire des images télévisuelles françaises de mai 68 à mai 2018, en passant par ce mai 1981, qui vit la victoire de la gauche et le début des années Mitterrand (Ory, 1983). Plusieurs étapes de l'histoire récente sont ainsi convoquées qui encadrent 
l'évolution du panorama audiovisuel français dans la foulée de Mai 68 - les « images » dont il est ici questions: les deux chocs pétroliers, mais surtout l'ascension mitterrandienne : "Le soir du 10 mai, Jérôme et Sylvie buvaient le champagne (...)» (Rémond, $1997:$ 38).

La stratégie narrative d'exhumation des souvenirs de Mai 68 de Camarades de classe est tout autre. Il s'agit de partir du présent et de revisiter le passé de Mai (et son contexte social) à la faveur des posts échangés sur le forum camarades.de.classe.com (Daeninckx, 2008 : 16). En effet, le contenu, parfois acerbe, des différents témoignages suscités par la photo de groupe postée sur ce site, reconstitue le contexte descriptif de cette génération soixante-huitarde de la ceinture nord communiste de Paris, que le temps et les tendances ont complètement transformée du point de vue urbanistique, comme le signale Michel Lazano, un ancien camarade de classe. On est passé des usines métallurgiques aux cités et, plus tard, à un espace urbain alternatif et à vocation artistique (ibid. : 146). Les intervenants ne manqueront pas de signaler ironiquement à présent leur détachement du communisme, voire leur vision quasi muséologique de cette idéologie (ibid. : 45).

Si les retrouvailles finales des anciens " camarades de classe " pour une photo mise à jour quatre décennies plus tard ne représente pas vraiment ce vers quoi le récit tend, c'est parce que le propos est tout autre: croiser des impressions - pas seulement nostalgiques - de ces années soixante, et mesurer l'évolution des mentalités et des profils sociaux.

26 Ainsi, les intervenants sur le forum brossent le portrait sociologique d'une époque et d'une région : «Non, mais je continue à me souvenir d'où je viens. On ne bougeait pas, à l'époque, comme si notre condition sociale nous assignait à résidence » (ibid. : 29). En tant que « mômes de prolos » (ibid. : 30) - « Mon père, lui, était métallo, fraiseur chez Babcock et Willcox, à La Courneuve, avec un contremaître qui chronométrait ses moindres faits et gestes » (ibid. : 34) - pour qui l'ascension ou la simple mobilité sociale était interdite, ces ex-camarades craignaient le pire: devoir perpétuer les emplois subalternes et mécaniques de leurs parents, ne pas quitter l'usine : "J'ai compris ce que ça voulait vraiment dire, travailler à la chaîne... J'allais repartir quand elle [la mère] a senti une présence derrière elle (...) » (ibid. : 118). Ces aveux mettent à mal plusieurs clichés sur les Trente Glorieuses.

27 À cet égard, Ludivine Bantigny rappelle qu'« il faudrait remettre en question la notion même de Trente Glorieuses. En 1968, on entre à l'usine à 16 ans et on travaille 48 heures par semaine " (ibid. : 24). Mais subtilement, ce contexte passé est violemment mis en contraste avec un présent autrement implacable. En effet, François subit de plein fouet les plans sociaux (ibid.: 11) et les « ajustements » (ibid. : 80) en résultat d'un capitalisme sauvage qu'il associe à la logique des marchés de capitaux et à l'ascension du néolibéralisme : «'Il va comprendre'! Tu en as de bonnes... Qu'est-ce que tu crois qu'il va faire? Tu l'imagines en train de téléphoner aux goldens boys des fonds de pension de Floride, de Californie, pour les convaincre de se montrer prévenants, de respecter la fragilité de la nature humaine ? » (Ibid. : 70), dans ce qu'Edgar Morin qualifie, face à Mai 68, justement, de « néolibéralisme comme nouvelle idéologie » (Morin, $2018: 11$ ).

Si, côté nostalgie, le lecteur, au moins quinquénaire, aura l'occasion de renouer avec les idoles yéyés des années soixante-dix (Daeninckx, 2008: 38) et avec l'esprit et le phénomène "Salut les copains" (ibid.: 65) qui les a mythifiées, évoqué par Morin (Morin, 2018: 8), d'autres aspects permettent de complexifier, voire de nuancer les 
acquis de Mai 68, comme l'« école séparée » (Daeninckx, 2008: 51), les pratiques pédophiles hypocritement tolérées (Ambroise-Rendu, 2018:52) - notamment à l'école (Daeninckx, 2008: 62) -, la nouveauté de la contraception ou l'interdiction de l'avortement (ibid. : 120), pour ne pas parler de l'homosexualité, dont on apprend sur le site que François aurait sporadiquement pratiquée avec un camarade de classe. Ils démontent des idées reçues sur Mai 68 et rendent compte de ce que Michel Bozon qualifie de "lenteur des progrès" dans «La véritable histoire de la "libération sexuelle'" (Bozon, 2018: 48-51). Si « La vie sexuelle contemporaine a tout bonnement cessé de s'identifier à l'expérience du couple marié indissoluble et hétérosexuel. Un élargissement du cadre de la sexualité inimaginable pour les jeunes gens de 1968 » (ibid. : 50), tel n'est plus le cas quarante ans plus tard, et l'opération de changement de sexe de Dominique l'atteste.

Mais Camarades de classe est aussi l'occasion de replacer le curseur de l'Histoire sur les événements de Mai 68 proprement dits. Les souvenirs et les anecdotes personnels de ces participants sur cette époque bouillonnante sont encore vifs : « Le mal du pays m'a saisi en découvrant les images des barricades parisiennes sur l'écran de ma télé (...). Ils avaient laissé la place à la France profonde, à la France respectueuse (...) » (Daeninckx, 2008 : 44). Très symptomatiquement, un des intervenants signale trois moments ou soubresauts "euphoriques" comme balises contradictoires de l'évolution sociétale française : la Guerre d'Algérie, Mai 68 et les émeutes des banlieues en 2005, qui devaient expliquer l'ascension de Sarkozy et le virage à droite de la France profonde (ibid. : 157-158).

30 Tout comme dans Les Images, la solidarité collective et l'engagement spontané de jadis donnent à présent lieu à certaine mauvaise conscience ou à la tentation de se renier au travail comme dans la vie :

J'ai vécu moi aussi ces quelques mois irréels où la jeunesse d'une ville faisait corps, je me suis retrouvé dans cet enthousiasme qu'évoque Edgar Bernot, même si les luttes politiques me sont passées au-dessus de la tête. Seule comptait alors cette immense générosité qui nous animait (ibid. : 114-115).

31 Ne propose-t-on cyniquement pas à François de dresser la liste des emplois de son laboratoire à éliminer pour sauvegarder le sien (ibid. : 138)?

De notre brève analyse, il appert que le «roman de Mai » demeure un indicateur possible, et apporte un certain éclairage sur les mutations sociétales subies par la France depuis les années soixante, parfois mises sur le seul compte de Mai 68, ainsi qu'un pertinent recueil mémoriel - fictionnalisé, certes - de cette «brèche » ou de cette « extase » collective. D'autant plus que, cinquante ans plus tard, Mai 68 n'a toujours pas livré toutes ses lectures - loin s'en faut - comme le montrent plusieurs dossier thématiques et critiques parus à l'occasion du cinquantenaire ; ce qui fait accroire à une " confusion des interprétations " (Zancarini-Fournel \& Artières, $2018:$ 38-43). Raison pour laquelle un récent sondage publié et analysé pour Le nouveau Magazine Littéraire, 2018, n 3 par Emmanuel Poncet, - Sondage « De mai pense ce qu'il te plaît » (Poncet, 2018: 36-39) - laissait entendre que «(...) les meilleurs avocats de Mai 68 sont ceux... qui ne l'ont pas vécu » (ibid. : 39). Mais tous s'accordent à présent pour imputer à Mai 68 le "présentisme", cette "propension des sociétés contemporaines à vivre dans le présent, sans souci du passé ni de l'avenir (...) » (Bantigny, 2018: 25). Affaire à suivre, donc. 


\section{BIBLIOGRAPHIE}

AMBROISE-RENDU, Anne-Claude (2018). « L'apologie de la pédophilie, face noire de 68 », Les hors-série de l'OBS. « 68 : le grand tournant », nº 98, p. 52.

BANTIGNY, Ludivine (2018). « Mai 68 par-delà les clichés », entretien avec Éric Aeschimann, Les hors-série de l'OBS. « 68 : le grand tournant », no 98, pp. 22-25.

BAUDRILLARD, Jean (1981). Simulacres et simulation. Paris : Galilée.

BOzon, Michel (2018). « La véritable histoire de la 'libération sexuelle'«, Les hors-série de l’OBS.

« 68 : le grand tournant », no 98, pp. $48-51$.

BRENDLÉ, Chloé (2018). « L'introuvable récit », Le nouveau Magazine Littéraire, mars 2018, nº 3, pp. $46-48$.

DAENINCKX, Didier (2008). Camarades de classe. Paris : Gallimard, « Folio ».

FERRY, LuC \& RENAUT, Alain (1988). La Pensée 68. Essai sur l'anti-humanisme contemporain. Paris : Gallimard.

GAUTHIER, Ursula (2018). « Une extase de l'Histoire », Les hors-série de l'OBS. « 68 : le grand tournant », nº 98, p. 3.

Huston, Nancy (2004). Professeurs de désespoir. Paris : Actes Sud.

LE BRIS, Michel (2018). « De Mao à King Kong », Le nouveau Magazine Littéraire, mars 2018, ํㅡ 3, pp. 50-53.

LIPOVETSKY, Gilles (1988). L'Ère du vide. Essais sur l'individualisme contemporain. Paris : Gallimard.

LISARELLI, Diane (2018). « Je n'ai pas fait 68 mais... », Le nouveau Magazine Littéraire, mars 2018, ํㅜ 3, pp. 40-41.

MORIN, Edgar (2018). « Introduction », Les hors-série de l'OBS. « 68 : le grand tournant », nº 98, p. 6-13.

ORY, Pascal (1983). L'entre-deux-mai. Paris : Seuil.

ORY, Pascal (2018). « Une révolution dans l'expression », entretien avec Charles Giol, Les hors-série de l’OBS. « 68 : le grand tournant », nº 98, pp. 68-71.

PEREC, Georges (1965). Les Choses. Paris : Julliard, « Lettres nouvelles ».

PONCET, Emmanuel (2018). « De Mai pense ce qu'il te plaît », Le nouveau Magazine Littéraire, mars 2018, no 3, pp. 36- 39 .

RÉMOND, Alain (1997). Les Images. Paris : Seuil, « Points ».

WEBER, Henri (2008). Faut-il liquider Mai 68 ?, Paris : Seuil.

ZANCARINI-FOURNEL, Michelle \& ARTIÈRES, Philippe (2018). « La confusion des interprétations », entretien avec Éric Aeschimann, Les hors-série de l'OBS. « 68 : le grand tournant », no 98, pp. 38-43.

Sitographie

https://www.babelio.com/liste/1324/Mai-68 [consulté le 18/09/2018]

http://publictionnaire.huma-num.fr/notice/soixante-huitards/ [consulté le 18/09/2018] 


\section{NOTES}

1. https://www.babelio.com/liste/1324/Mai-68

\section{RÉSUMÉS}

Les romans Les Images d'Alain Rémond (1997) et Camarades de classe (2008) de Didier Daeninckx brossent le portrait narrent le cheminement erratique de toute une génération amplement marquée par les événements et les idées majeurs de Mai 68, mais qui a du mal à en assumer tout l'héritage ou à lui rester fidèle. Ainsi, c'est à un certain portrait de la France contemporaine, avec ses valeurs, ses contradictions et ses incohérences que nous avons affaire. Il s'agit d'une génération souvent fort éloignée des engagements sociopolitiques pris, et des slogans libertaires scandés lors de Mai 68. Ce bilan ambivalent est notamment dressé par le biais de visions et de (re)lectures contradictoires de ce même passé de moins en moins « récent ».

The novels Les Images by Alain Rémond (1997) and Camarades de classe (2008) by Didier Daeninckx paint the portrait - sometimes difficult to recognize - and narrate the erratic path of a generation amply marked by the events and major ideas of May 68 but who find it difficult to assume its whole legacy or to remain faithful to it. In doing so, it is to a certain portrait of contemporary France, with its values, its contradictions and its inconsistencies that we are dealing, and with a generation often far removed from the socio-political commitments made, and libertarian slogans chanted during May 68. This ambivalent report is drawn up namely through contradictory visions and (re)readings of this less and less "recent" past.

\section{INDEX}

Keywords : May 68, contemporary novels, Rémond (Alain), Daeninckx (Didier)

Mots-clés : Mai 68, romans contemporains, Rémond (Alain), Daeninckx (Didier)

\section{AUTEUR}

\section{JOSÉ DOMINGUES DE ALMEIDA}

Un. Porto - APEF - ILC ML

jalmeida[at]letras.up.pt 